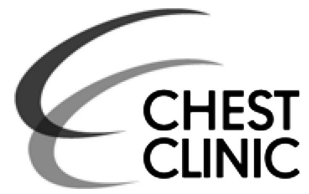

${ }^{1}$ Center for Medical Genetics, Ghent University, Ghent, Belgium

${ }^{2}$ COPD Translational Laboratory, Department of Respiratory Medicine, Ghent University Hospital, Ghent, Belgium

${ }^{3}$ Department of Epidemiology and Respiratory Medicine, Erasmus Medical Center Rotterdam, Rotterdam,

The Netherlands

${ }^{4}$ Tumor Immunology Laboratory, Department of Respiratory Medicine, Ghent University Hospital, Ghent, Belgium

\section{Correspondence to}

Professor Karim Vermaelen,

Tumor Immunology Laboratory, Department of Respiratory

Medicine, Ghent University

Hospital 7K12ie, De Pintelaan

185, Ghent B-9000, Belgium;

Karim.Vermaelen@ugent.be

Received 3 October 2014 Accepted 16 October 2014 Published Online First

5 November 2014

\title{
Non-coding RNAs and respiratory disease
}

\author{
Pieter Mestdagh, ${ }^{1}$ Jo Vandesompele, ${ }^{1}$ Guy Brusselle, ${ }^{2,3}$ Karim Vermaelen $^{4}$
}

\section{ABSTRACT}

Recent scientific developments have radically changed the way we look at the vast 'non-coding' part of our genome. It is now clear that this genomic 'dark matter' is transcribed into myriads of RNA species that act behind the scenes to veto, or boost, the production of proteins in our cells. As a consequence, non-coding RNAs (ncRNAs) represent an additional layer of regulation for fundamental biological processes such as organ development, tissue repair and immunity. It also follows that disturbances in ncRNA networks (among which microRNAs and long ncRNAs are the best studied) can give rise to a whole range of pathological conditions. Increasing preclinical and translational evidence places ncRNAs as key players in a wide spectrum of diseases affecting the lung. In this concise review, we will provide essential concepts of ncRNA science, with special emphasis on discoveries relevant to the pulmonary physician.

\section{INDISPENSABLE JUNK}

Until recently, it was believed that only a small fraction of the genome contained relevant information, defined as instructions for the cell to produce proteins. The majority was referred to as 'junk DNA' with no obvious function throughout life. Today, there is insurmountable evidence demonstrating that the human genome is pervasively transcribed, giving rise to tens of thousands of so-called noncoding RNA (ncRNA) transcripts. Unlike messenger RNAs, ncRNAs do not serve as template for protein synthesis, but instead play a major role in regulating protein-coding gene expression. The ncRNA repertoire consists of small ncRNAs such as microRNAs (miRNAs, 18-24 nucleotides in length) and long ncRNAs (lncRNAs, >200 nucleotides), both of which have important functions in various aspects of cell biology. miRNAs have been most thoroughly investigated, function as negative regulators of gene expression and are predicted to target as much as $60 \%$ of all human protein-coding genes. Perturbed miRNA expression has been reported for nearly all human diseases and several miRNA-based therapeutics are entering clinical trials. LncRNAs, on the other hand, are far less characterised compared with miRNAs, but they comprise the bulk of the non-coding transcriptome. In contrast to miRNAs, lncRNAs can either repress or boost the expression of proteins. Additionally, while miRNA function is highly conserved across different species, IncRNA function seems to be more specific in terms of species, tissue and developmental stage. Many of these lncRNAs are associated with disease-linked genetic variations, hinting at a possible role in human disease and development. A growing number of studies provide evidence that lncRNA expression is deregulated in human disease and that IncRNA perturbation impacts on various disease phenotypes. The potential importance of ncRNAs in development is further supported by the intriguing observation that organism complexity (as defined by the number of different cells) is intimately linked to the proportion of the genome that is non-coding. ${ }^{1}$ In contrast to the protein-coding portion of the genome, which is virtually similar in size in fruit flies and humans, the non-coding counterpart increases in size with increasing organism complexity. Here, we highlight some of the key aspects of ncRNAs in lung disease, either as biomarker or as therapeutic target.

\section{NCRNAS IN LUNG BIOLOGY AND DISEASE}

A key task of ncRNAs appears to be the regulation of cell and organ homeostatic processes, including proliferative responses as required for tissue repair, as well as inflammatory/immune responses. It follows that an imbalance in the ncRNA system leads to the pathological counterparts of these homeostatic mechanisms, which for the respiratory system translates into lung cancer, COPD/emphysema, lung fibrosis or chronic infection.

COPD and lung fibrosis both arise in a context of chronic tissue injury and aberrant repair, and both constitute fertile grounds for lung tumorigenesis. The link between chronic inflammation and carcinogenesis is now widely acknowledged and is considered as one of the 'enabling hallmarks' of cancer. There is ample evidence that ncRNAs are situated at the core of this association (figure 1). Several miRNAs are implicated in aberrant immune/inflammatory responses as well as oncogenesis. The let-7 miRNA gene family has been well documented as having tumour-suppressive functions in lung cancer. Decreased expression of let-7 in the sputum of patients with COPD has fuelled the speculation that a combination of chronic airway inflammation together with the loss of a major tumour suppressor may contribute to the increased risk of patients with COPD to develop lung cancer. In addition to miRNAs, lncRNAs have also been identified with tumourpromoting and tumour-suppressing functions in lung cancer. ${ }^{2}$ MALAT1 is the first characterised lncRNA in lung cancer and has been shown to be a powerful predictor of metastatic relapse in patients with non-small cell lung cancer. SCAL1 is an lncRNA whose expression rises in airway epithelial cells as part of the oxidative stress response to cigarette smoke exposure. SCAL1 is also overexpressed in a whole range of lung cancer cell lines, 


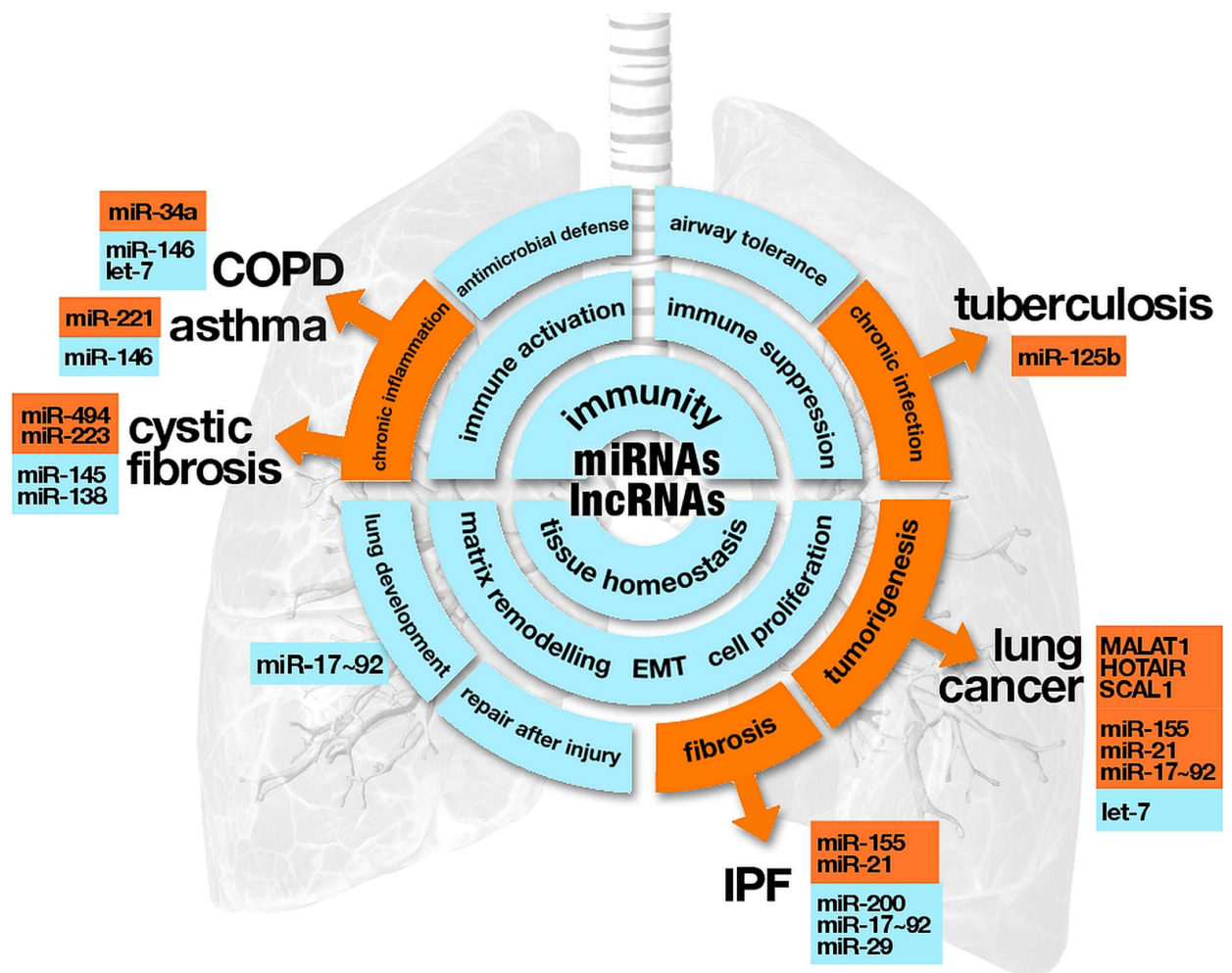

Figure 1 Non-coding RNAs (ncRNA) regulate core processes underlying organ development and safeguarding of tissue integrity. Highlighted (orange) are the pathological entities in which these homeostatic processes fail, along with the corresponding disease manifestations in the lung. For each pulmonary disease entity, a (non-exhaustive) list with some of the major implicated microRNA (miRNA) and long non-coding (IncRNA) is indicated (orange: overexpressed in the disease; blue: repressed). The diagram illustrates some degree of overlap in miRNA network deregulation among airway inflammatory diseases (COPD, asthma). Also, common patterns of aberrant miRNA expression emerge when comparing lung fibrosis and lung cancer, and to some extent lung development (eg, miRNAs from the miR-17-92 cluster are normally silenced after early embryonic lung development, while persistent overexpression of these miRNAs disrupts lung morphogenesis and is associated with lung tumorigenesis). MALAT1 $\left(\right.$ Inc-SCYL1 ${ }^{*}$ ), HOTAIR (Inc-SMUG1-7*) and SCAL1 (Inc-ARRDC3-1*) are the best described oncogenic IncRNAs in lung cancer (* LNCipedia database nomenclature, http://www.Incipedia.org). EMT, epithelial to mesenchymal transition; IPF, idiopathic pulmonary fibrosis.

possibly pointing to a novel mechanism of smoke-induced malignant transformation.

Idiopathic pulmonary fibrosis (IPF) shares several pathobiological features with cancer, among which epithelial to mesenchymal transition (EMT) is best documented. In cancer, EMT is a critical process underlying invasive behaviour, while in IPF, EMT drives the transdifferentiation of alveolar epithelial cells into a network of infiltrating myofibroblasts. The sharing of this pathological pathway is in line with the classical view on cancer as 'a wound that never heals'. Not surprisingly, a fairly consistent finding in preclinical models of lung fibrosis and in lung tissue samples of patients with IPF is the loss of specific miRNAs that repress EMT such as miR-200.

In chronic airway inflammatory diseases such as asthma and COPD, studies on lung tissue, sputum and serum of patients have revealed associations with specific patterns of miRNA expression. A recurrent theme is the loss of anti-inflammatory miRNA activity (eg, decrease of miR-146). We would like to refer the reader to several excellent reviews that summarise the large body of data on the subject. ${ }^{3}$

Cystic fibrosis (CF) is also dominated by a state of chronic, destructive airway inflammation, and recent studies on patient samples have revealed CF-specific perturbations in miRNA expression. Moreover, there is evidence that ncRNAs could be implicated in CF pathogenesis: in vitro tests have shown that replenishment of an miRNA that is specifically lost in CF airways has the potential to boost functional cystic fibrosis transmembrane conductance regulator (CFTR) protein synthesis and restore transmembrane conductance. Conversely, inflammatory responses drive the expression of miRNA species that repress CFTR protein abundance.

\section{NCRNAS: READY FOR PRIME TIME AS LUNG DISEASE BIOMARKERS?}

In clinical practice, disease biomarkers can be used for different objectives: (1) screening, which is the most controversial implementation and imposes high standards in terms of sensitivity and specificity; (2) disease classification (eg, asthma phenotypes, lung cancer subtypes); (3) prognosis (prediction of survival outlook regardless of therapy); (4) theragnosis (prediction of response to a specific treatment) and (5) therapeutic monitoring. Technically speaking, miRNAs are particularly attractive as potential biomarkers given their stability in the blood circulation (and in other body fluids such as saliva, sputum and urine), which may stem from the fact that circulating miRNAs are either bound to protective protein complexes or packaged in small secreted vesicles called exosomes, protecting them from degradation. Of note, confounding factors (eg, systemic inflammation in patients with cancer) may hamper test specificity of the biomarker signature.

In pulmonary medicine, most of the ncRNA-related biomarker research has focused on miRNAs in lung cancer, specifically in the context of screening and diagnosis. More than 1000 papers have been published in this field, yet no single 
miRNA or miRNA signature has passed the test as an analytically validated and clinically useful biomarker. Attempts have been made through meta-analysis to assess the diagnostic accuracy of miRNAs in lung cancer early diagnosis. ${ }^{4}$ The largest published systematic review established the superiority of combined miRNA signatures versus single miRNA species, with an overall estimate of sensitivity of $0.81(0.75-0.86)$, specificity 0.84 $(0.80-0.86)$ and an area under the curve of 0.89 on the receiver operating curve. Similar to what has been observed for mRNA biomarker signatures, there is little overlap between the miRNAs discovered in these studies. However, it is not excluded that these different miRNAs in fact regulate common biological pathways. It remains to be seen whether IncRNAs, with their more restricted tissue-specific expression pattern, will be superior in terms of specificity.

\section{FUTURE OPPORTUNITIES: NCRNAS AS THERAPEUTIC TARGETS IN LUNG DISEASE}

Targeted inhibition of miRNA activity in vivo has proven to be a successful approach in preclinical models for a wide range of diseases. Effects are achieved by injecting synthetic short nucleotide sequences ('antagomirs') that are complementary to and sequester-specific miRNA species, preventing them from repressing protein expression. Antagomirs are chemically modified to increase in vivo stability and half-life. This approach has now moved beyond the proof of concept: the recent demonstration that inhibiting a liver-specific miRNA in hepatitis $C$ virusinfected patients can produce long-lasting clinical benefit can be considered a significant advancement in medicine as it will hopefully open the way for more miRNA-targeted therapies in other chronic diseases. ${ }^{5}$ Despite the pleiotropy of targets under control of a single miRNA species, the compound used in the trial (miravirsen, an antagomir targeting miR-122) showed a remarkably low-toxicity profile considering it was delivered systemically. Low toxicity can, in part, also be explained by the tissue-specific nature of ncRNA expression. In that respect, lungspecific ncRNAs constitute attractive targets for therapeutic inhibition. The lung also offers a unique opportunity for local delivery of miRNA-targeting agents, allowing direct targeting of structural and/or immune pulmonary compartments with lower compound doses. Obviously, issues pertaining to the formulation of an 'inhaled antagomir' in terms of optimal stability and lung deposition have to be addressed, but should be within technical reach. Still, a possible flipside is that exogenous RNA sequences can be potent triggers of innate immune receptors, which, in the pulmonary environment, could result in potentially severe inflammatory reactions. An even greater challenge remains the identification of adequate targets: preclinical and translational research efforts in ncRNAs should first tell us whether an observed perturbation of an ncRNA in a lung disease is a mere epiphenomenon or represents the actual driving force behind the disorder. Nevertheless, the payoff is enormous as these efforts could pave the way for a new generation of therapies in asthma, COPD, lung cancer, IPF and CF, all incurable conditions that constitute a massive burden on global health.

Contributors PM, JV, GB and KV all contributed to the conception of the review, the literature search, the manuscript drafting and its revision. In addition, all the listed coauthors approve the version to be published and are accountable for the accuracy and integrity of the work. KV was responsible for producing the illustration.

Funding Concerted Research Initiative of Ghent University—BOF. GOA.2014.0008.01.

Competing interests None.

Provenance and peer review Not commissioned; internally peer reviewed.

\section{REFERENCES}

1 Liu G, Mattick JS, Taft RJ. A meta-analysis of the genomic and transcriptomic composition of complex life. Cell Cycle 2013;12:2061-72.

2 White NM, Cabanski CR, Silva-Fisher JM, et al. Transcriptome sequencing reveals altered long intergenic non-coding RNAs in lung cancer. Genome Biol 2014;15:429.

3 Booton R, Lindsay MA. Emerging role of microRNAs and long noncoding RNAs in respiratory disease. Chest 2014;146:193-204.

4 Guo Z, Zhao C, Wang Z. MicroRNAs as ideal biomarkers for the diagnosis of lung cancer. Tumour Biol 2014;35:10395-407.

5 Janssen HLA, Reesink HW, Lawitz EJ, et al. Treatment of HCV infection by targeting microRNA. N Engl J Med 2013;368:1685-94. 